Clío Américal Vol. 12, No. 24 - 2018 / 122 - 136

DOI: http://dx:10.21676/23897848.2650

\title{
COMPETENCIAS DE LOS DIRECTIVOS DOCENTES PARA LA TRANSFORMACIÓN DE LAS INSTITUCIONES EDUCATIVAS EN ORGANIZACIONES ESCOLARES INTELIGENTES
}

\author{
COMPETENCIES OF TEACHING DIRECTORS FOR THE TRANSFORMATION OF \\ EDUCATIONAL INSTITUTIONS INTO INTELLIGENT LEARNING ORGANIZATIONS
}

\section{Jorge Oswaldo Sánchez-Buitrago ${ }^{1}$, Johanna Cintia Rúa-Martínez ${ }^{2}$ y Loly Esther Ternera- Romero $^{3(1)}$}

Tipología: Artículo de investigación científica y tecnológica

Para citar este artículo: Sánchez, B.J., Rúa, M.J. y Ternera, R.L. (2018). Competencias de los directivos docentes para la transformación de la institución educativa en una organización escolar inteligente. Clío América, 12(24), 122 - 136. doi: http://dx.doi.org/10.21676/23897848.2650

Recibido en julio 22 de 2018

Aceptado en 07 de noviembre de 2018

Publicado en línea en 28 de noviembre de 2018

\begin{abstract}
El objetivo del presente artículo es caracterizar las competencias para la gestión educativa de los directivos docentes del departamento del Magdalena desde una reflexión sobre sus implicaciones para la transformación de las instituciones educativas en organizaciones escolares inteligentes. En este sentido, se desarrolló una investigación mixta de tipo descriptiva, que privilegió diferentes herramientas de recolección de información como una Matriz de Análisis de Hojas de Vida de los directivos docentes, para identificar las variables sociodemográficas de los mismos, y un Cuestionario para evaluación de competencias para la gestión educativa de los directivos docentes. Adicionalmente, se desarrollaron entrevistas, grupos focales y análisis documental para profundizar la información recolectada en los instrumentos aplicados. En términos de resultados, los directivos docentes expresan, en la mayoría de los casos, que tienen desarrolladas las competencias para la gestión de las instituciones educativas, pero esto no se evidencia en los resultados en términos de evaluación de la calidad que aplican en el orden territorial. A partir de estos hallazgos se concluye que los rectores y coordinadores deben desarrollar los procesos de gestión de una manera crítica, sistemática y reflexionada desde las implicaciones de la resignificación para transformar las instituciones educativas en organizaciones escolares inteligentes.
\end{abstract}

Palabras clave: Competencias - Gestión educativa - Instituciones educativas - Organizaciones escolares inteligentes - Resignificación. JEL: I2, I23, I25, I28

\section{ABSTRACT}

The objective of this article is to characterize the competencies in education management of the teaching directors of the department of Magdalena, by reflecting on the impact they have on the transformation of educational institutions into intelligent learning organizations. In this sense, a mixed-descriptive research was developed. It privileged different information gathering tools: a Matrix of Analysis of the curriculum vitae of several educational managers in order to identify their sociodemographic variables, and a Questionnaire to evaluate the competences of teaching directors in educational management. Additionally, interviews, focus groups and documentary analysis were developed to deepen the information collected with the tools mentioned above. In terms of results, the teaching executives expressed that, in most cases, they have developed competencies for the management of educational institutions, but this is not evident in terms of quality assessment at the regional level. From these findings, it is possible to conclude that rectors and coordinators must develop the management processes in a critical, systematic and thoughtful way, taking the implications of resignification into account, in order to transform educational institutions into intelligent learning organizations.

Keywords: Competencies - Educational management - Educational Institutions - Smart school organizations - Resignification.

1 Universidad del Magdalena, Santa Marta, Colombia. Email: joswaldosanchez@unimagdalena.edu.co ORCID: https://orcid.org/0000-0002-9299-6647

2 Institución Educativa Departamental Las Mercedes, Zona Bananera, Colombia. Email: jrua99@ hotmail.com ORCID: https://orcid.org/0000-0001-95099768

3 Institución Educativa Departamental Las Mercedes, Zona Bananera, Colombia. Email: lolyluz21@hotmail.com ORCID: https://orcid.org/0000-00031790-8077 


\section{COMPETENCIAS DE LOS DIRECTIVOS DOCENTES PARA LA TRANSFORMACIÓN DE LAS INSTITUCIONES EDUCATIVAS EN ORGANIZACIONES ESCOLARES INTELIGENTES}

\section{INTRODUCCIÓN}

Los procesos de globalización de las sociedades actuales han generado dinámicas de transformación de los sistemas y las organizaciones (Daza, Miranda y Rodríguez, 2017). A partir de lo anterior, el sistema educativo en Colombia ha sido diseñado para dar respuesta a los indicadores establecidos en la evaluación de la calidad educativa en las instituciones escolares, lo que Perkins (1995; 2000) denomina inteligencia de laboratorio. En este sentido, los directivos docentes se han interesado en enfocar los procesos de gestión hacia el cumplimiento de tales indicadores, desconociendo en muchas ocasiones la complejidad de las organizaciones educativas (Sánchez, Linero y Martínez, 2014; Liñán y Polo, 2017). El departamento del Magdalena no es ajeno a esta dinámica y su situación se complica al presentar resultados negativos en los mecanismos formales de valoración educativa que utiliza el Ministerio de Educación Nacional.

Aunque la gestión de las organizaciones educativas es un tema que ha merecido especial atención por diversos investigadores y los organismos encargados del control de la política al respecto, esta siempre ha estado enfocada en unos criterios procedimentales y mecanicistas de los procesos institucionales y no en los elementos pedagógicos que dotan de sentido a las instituciones (González, 2017), por tanto, se encuentra alienada a una disposición tradicional de intentar explicar las dinámicas de las organizaciones sin explorar su universalidad y complejidad (Peralta, 2016). Al asumir las organizaciones educativas como construcciones sociales que se componen de unas asociaciones formales e informales (Sánchez, Viloria y Miranda, 2017), toma mayor relevancia el proceso de gestión que desempeñan los directivos docentes (rectores y coordinadores) y, en consecuencia, las competencias que han desarrollado para dicha gestión.

El ejercicio de la gestión por parte de los directivos docentes es fundamental en el proceso de transformación de una institución educativa a una organización escolar inteligente, considerando que las competencias y el nivel de formación de los docentes tiene una incidencia significativa en el funcionamiento y calidad de las organizaciones educativas (Torres, Vélez y Altamar, 2015). La institución ha sido construida a partir de las definiciones legales, formuladas por la legislación educativa, y desarrollada a través de los procesos de planeación educativa y del ejercicio del control por parte de las instancias que conforman el sistema educativo nacional. La organización escolar inteligente centra sus objetivos en el aprendizaje de los estudiantes y en el logro de metas relacionadas con éste, para ello, estimula el trabajo en equipo por parte de quienes integran la comunidad educativa de cada organización, el diálogo, la integración de los procesos que involucran los componentes de gestión, a saber: pedagógica, administrativa - financiera, directiva y comunitaria, porque de ellos depende el fortalecimiento y la mejora progresiva de los aprendizajes y, desde luego, de la calidad. Pero entendiendo a la calidad desde una perspectiva multifactorial que depende de las concepciones de educación que se tengan y de las necesidades del contexto (Viloria, Bertel y Daza, 2015).

Además, el liderazgo de los directivos docentes es fundamental en la transición de la institución a la organización, porque mediante el direccionamiento efectivo y la interacción entre los diferentes procesos involucrados en la gestión educativa se avanza en la construcción y el desarrollo de la organización (Rodríguez, 2017a, 2017b).

En este sentido, se entiende a las organizaciones educativas como un sistema que se construye socialmente, en la cual se requiere una interacción de los actores para develar su realidad, debido a la misma complejidad que las rige, por tanto, cada organización educativa difiere una de otra, en el sentido que cada una desarrolla un diálogo entre de lo instituido y lo instituyente de manera diferente, lo que conlleva a que estas no pueden ser tratadas bajo un mismo esquema, cada una es única y sus vivencias son particulares (Sánchez, 2014).

La gestión de ese sistema de complejidades y las prácticas enfocadas en su transformación hacia una organización escolar inteligente requiere de un conjunto de competencias por parte de los directivos 
docentes. En este sentido, Ocampo, Castro, Becerra y Herrera (2014) sostienen que la competencia es la habilidad de actuar críticamente frente a diversas situaciones, permitiendo el intercambio de conocimientos y la solución creativa de problemáticas mediante la utilización de diferentes recursos cognitivos. En ese sentido, Higuita, Molano y Rodríguez (2011) dicen que las competencias son los atributos personales que permiten la diferenciación en los grados de desempeño y las formas en que se desarrollan las actividades, haciendo énfasis en las habilidades y comportamientos para lograr el éxito en los diversos escenarios. En general, las competencias han sido asociadas a las capacidades que tienen las personas para resolver las diversas problemáticas que se presentan en sus dinámicas cotidianas (González, 2006).

Por su parte, el término de gestión tradicionalmente ha sido asociado al escenario empresarial, pero en los últimos años se ha llevado sus contenidos teóricos y prácticos a los procesos de direccionamiento de las instituciones educativas (Correa, Álvarez y Correa, 2012). Según Sánchez, Sánchez y Viloria (2017):

La gestión educativa se convierte entonces en un asunto de planeación, ejecución, organización y verificación que integra a cada uno de los componentes de la organización en torno a las significaciones e identidades que se han construido para encadenar los procesos administrativos (p. 35-36).

La gestión educativa debe asumirse como una praxis comprensiva y transformadora que está siempre guiada por una intencionalidad teleológica, es decir, en función de finalidades de desarrollo integral de alta calidad. En concordancia con lo anterior, las competencias requeridas por los directivos docentes para el proceso de gestión y transformación de las instituciones educativas en organizaciones escolares inteligentes deben estar alineadas a la configuración de sistemas flexibles, que no solamente sean capaces de acomodarse a retos que emergen del contexto sino que hagan aprovechamiento de tales dinámicas para su transformación (Senge, 1990; Aguerrondo, 1998) $y$, privilegien el enfoque humanista en los procesos de direccionamiento (Drucker, 2012).

La importancia del artículo se evidencia en el recorrido que realiza por las competencias de los directivos docentes en el departamento del Magdalena, reflexionando acerca de las habilidades requeridas para la gestión y transformación de las instituciones educativas en organizaciones escolares inteligentes, que respondan a las necesidades sociales internas y a las realidades del territorio, en perspectiva del mejoramiento de las condiciones de vida de los habitantes, ya que, según Silva (2017) y Lombana y Muñoz (2017), existe una relación entre educación y competitividad en las regiones.

En este orden de ideas y considerando las similitudes del contexto territorial con otros escenarios geográficos y sociales, se pueden plantear argumentos generalizadores para la resignificación de las prácticas de gestión de las organizaciones educativas, asumidas estas como sistemas sociales complejos donde subyacen unos elementos instituidos e instituyentes (Sánchez, Chica y Viloria, 2017; Polo y Liñán, 2017).

\section{METODOLOGÍA}

En este apartado se presentan los principales encuadres metodológicos que se consideraron en el desarrollo investigativo.

Enfoque investigativo. La investigación se desarrolló desde un enfoque mixto en los términos de Hernández, Fernández y Baptista (2010). En este sentido, la información cuantitativa corresponde a las caracterizaciones sociodemográficas de los directivos docentes y la medición de las competencias para la gestión, y la cualitativa equivale a las voces de los actores sociales claves participantes.

En los alcances de la investigación cualitativa, el estudio también se ejecutó desde la perspectiva de la complementariedad propuesta por Murcia y Jaramillo (2000), al sostener que este enfoque permite que la investigación vaya configurando sus estructuras metodológicas en la medida que se 
progresa en el proceso investigativo.

Tipo de investigación. La investigación es de tipo descriptiva en las implicaciones que propone Ortiz (2015) cuando dice que "consiste en la determinación de las características de un evento de estudio" (p. 45). La categoría de su objetivo es describir, es decir, identificar, clasificar o caracterizar un evento. A partir de lo anterior, la descripción se realiza de las competencias para la gestión de los directivos docentes del departamento del Magdalena.

Unidad comprensiva. Corresponde a las 151 instituciones de educación básica y media de carácter público del departamento del Magdalena.

Actores sociales. Los sujetos objeto de estudio en la investigación fueron los 151 rectores de las instituciones educativas de básica y media del departamento del Magdalena y los 415 coordinadores de las mismas instituciones.

Instrumentos de recolección de información. Para el proceso de levantamiento de la información se utilizaron dos instrumentos inicialmente: primero, se elaboró una Matriz de Análisis de Hoja de Vida que se aplicó a los currículos de los rectores y coordinadores. Para la elaboración de este instrumento se tuvo como referencia el formato de Hoja de Vida la Función Pública definida por el Estado para todas las personas que tienen vínculos contractuales con el mismo. La validación de esta matriz se realizó a través de la aplicación de un pilotaje a tales documentos. Y por otro lado, se elaboró un Cuestionario para Evaluación de Competencias para la Gestión Educativa de los Directivos Docentes que pretendió identificar el nivel de desarrollo de las competencias de los directivos docentes. Adicionalmente, se desarrolló un proceso de análisis documental de las políticas educativas del contexto local y nacional. Así como también se aplicaron entrevistas y grupos de discusión para profundizar en la información recolectada a través de los instrumentos mencionados anteriormente.

Momentos de la investigación. En el marco de las implicaciones de la complementariedad propuesta por Murcia y Jaramillo (2000), la investigación se desarrolló en tres círculos metodológicos que se ejecutaron a manera de un gran círculo investigativo de primer orden. Los círculos investigativos fueron:

- Círculo 1 (C1): Fundamentación teórica de las categorías referenciales de la investigación.

- Círculo 2 (C2): Caracterización de las competencias para la gestión educativa de los directivos docentes del departamento del Magdalena.

- Círculo 3 (C3): Reflexión sobre la transformación de las instituciones educativas en organizaciones escolares inteligentes.

Dentro de cada uno de estos círculos se vivieron tres momentos metodológicos: preconfiguración, configuración y reconfiguración de la realidad.

La organización, tratamiento y análisis de la información recolectada durante el proceso investigativo se desarrolló a través de la utilización de las herramientas estadísticas de Microsoft Excel para los datos cuantitativos, y para los cualitativos se utilizó un protocolo de investigación que permitía agrupar las voces de los actores sociales de acuerdo a los círculos investigativos definidos.

\section{RESULTADOS}

Acercamiento al perfil sociodemográfico de los directivos docentes en el departamento del Magdalena

El directivo docente debe ser capaz de promover entre los miembros de la organización escolar a la que pertenece, la construcción de escenarios donde se posibilite la resignificación, entendida como la acción de dotarla de nuevos sentidos, y generar espacios para; cambiar la praxis discursiva de los docentes, estimular una postura crítica por parte de los alumnos hacia el aprendizaje, involucramiento por parte de los padres de familia y una comunicación con el entorno regional y nacional de manera efectiva, todo esto englobado en una concepción renovada de la práctica pedagógica 
(Sánchez, 2010).

En este sentido, desde una caracterización sociodemográfica encontramos que el 78,9\% de los participantes de la investigación identificados como directivos docentes en el cargo de Rector se encuentran por encima de los 45 años de edad, lo que imposibilita su ingreso a los programas de formación promovidos a través de becas por las entidades territoriales y nacionales. Además, un $11,3 \%$ ya se encuentran próximos a la edad de retiro.

En términos de una caracterización por género, se puede identificar que el porcentaje de participantes de sexo masculino es de $63,4 \%$ y del sexo femenino es de $36,6 \%$, encontrándose una relación donde el sexo masculino supera por casi el doble al femenino. Así mismo, el 71,8 \% de los rectores se encuentran nombrados en propiedad, sin embargo, se evidencia que el 7,0 \% de ellos están en asignación de funciones o en encargo el 21,1\%. A pesar de que se han dado procesos de concursos para proveer cargos de manera permanente, las vacantes no han sido provistas, lo que permitiría renovación del recurso humano. En este sentido, solo un 25,4\% pertenecen al régimen del decreto 1278 y un $74,6 \%$ son del decreto 2277.

En este mismo orden de ideas, se puede sostener que los docentes pertenecientes al decreto 1278 se encuentran ubicados en el nivel 2 en un 83,3\%. Solo seis (6) de los participantes evidencian especialización y uno (1) maestría, los demás solo tienen la formación profesional y se han reubicado por tiempo de servicio.

En lo que respecta a los directivos vinculados por medio del decreto 2277, un 32,1\% está ubicado por debajo de la categoría máxima que estipula este decreto, por lo que la formación implicaría una mejora salarial. El 67,9\% restante se encuentra en la categoría 14, por lo que la formación ya no implica cambios a nivel salarial, y su motivación para participar de procesos de formación respondería más al orden de expectativas y satisfacción de necesidades de formación para actualizarse y estar a tono con los procesos de competencias tan exigidos a los vinculados a través del decreto 1278 .

Por ser el departamento del Magdalena en su mayoría Rural, así el 65,2 \% laboran en esta zona, mientras que $34,8 \%$ lo hacen en zonas urbanas.

Continuando con el proceso de caracterización de directivos docentes en el cargo de Coordinador, se analiza que los encuestados que se encuentran por encima de los 45 años de edad corresponden al 60,6 $\%$. De los estos, un 9,3\% ya se encuentran próximos a la edad de retiro.

Por otro lado, se evidencia que el porcentaje de participantes de sexo masculino es del 60,5\% mientras que el $39,5 \%$ corresponde a mujeres. De los cuales se encuentran nombrados en propiedad el 65,1 $\%$ del total. Es importante resaltar que del global de coordinadores el $7 \%$ se encuentra en período de prueba, en asignación de funciones el $7 \%$ y en encargo el 20,9\%. Por otro lado, un 51,2 \% pertenecen al decreto 1278 y un $48,8 \%$ pertenecen al decreto 2277.

En cuanto a los coordinadores pertenecientes al decreto 1278, estos se encuentran en un 86,2 \% ubicados en el grado 2 en sus diferentes niveles, solo cinco (5) de los participantes evidencian especialización y tres (3) maestría, los demás solo tienen la formación profesional y se han reubicado por tiempo de servicio, cabe notar que algunos pese a que llevan los años requeridos para solicitar reubicación no lo han hecho debido a la difícil movilidad dentro de este nuevo estatuto, según lo expresado por algunos de los participantes.

En lo referente al decreto 2277, un 4,8 \% está ubicado por debajo de la categoría máxima de este decreto, por lo que la formación implicaría una mejora salarial, mientras el 95,2 \% está en la categoría 14, para este porcentaje de los participantes la formación responde más a intereses de cualificación laboral que a mejora salarial.

Hasta este punto, se han expuesto algunas de las características sociodemográficas de los directivos docentes en el departamento del Magdalena como punto de referenciación para la valoración del desarrollo de las competencias requeridas para la gestión de las organizaciones escolares.

\section{Caracterización del nivel de las competencias}




\section{para la gestión educativa de los directivos docentes en el departamento del Magdalena}

El directivo docente tiene el reto de orientar la organización y cada uno de sus integrantes hacia la consecución de unos objetivos y de unas metas comunes. Así pues, el directivo docente debe tener unas capacidades que le permiten asumir el ejercicio de las funciones propias del cargo y contribuir al fortalecimiento de la organización escolar inteligente. Además, como lo señala Correa (2005), la mirada del directivo como diseñador de una estrategia de largo plazo para la organización, será determinante para la estructuración de la visión de la organización y su conversión en un propósito de todos.

En este sentido, los directivos docentes deben tener un nivel de desarrollo significativo de las competencias para la gestión de las organizaciones escolares. En la figura 1 se detallan dichos niveles de desarrollos de acuerdo a las dimensiones requeridas para la transformación de las instituciones educativas en organizaciones escolares inteligentes.

Figura 1. Competencias para monitorear, anticipar y analizar

III Competencias para monitorear y evaluar los procesos en las organizaciones educativas

iㅣ Competencias para anticipar y proyectar los procesos de gestión de las organizaciones educativas

III Competencias para analizar y sintetizar los procesos de gestión en las organizaciones educativas

Poco desarrollada

Medianamente desarrollada
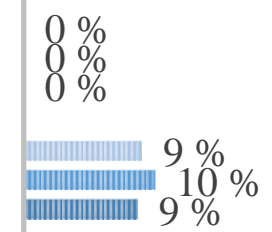

\section{Desarrollada}

Muy desarrollada

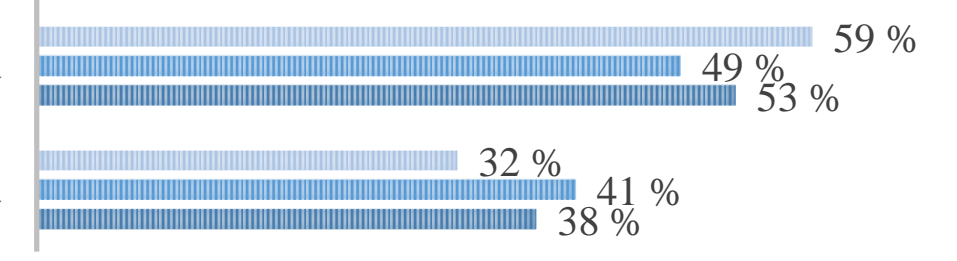

Fuente: elaboración propia

Inicialmente se exploran las competencias para analizar y sintetizar los procesos de gestión en las organizaciones educativas. En este sentido, el $19 \%$ de los directivos consideran que tiene muy desarrollada la competencia de aplicación de las directrices institucionales para garantizar el funcionamiento de los procesos de la institución, un $67 \%$ afirma tener una competencia desarrollada mientras que un $14 \%$ cree que la tienen medianamente desarrollada.

Por su parte, en la competencia de aplicación de las directrices públicas relacionadas con la administración de instituciones educativas; el $43 \%$ de los directivos la tienen muy desarrolla, el $52 \%$ desarrollada y un $5 \%$ dice tenerla medianamente desarrollada. En cuanto a la competencia del conocimiento y aplicación de la normatividad relacionada con el uso de recursos públicos en las instituciones educativas, el $57 \%$ dice que la tienen muy desarrollada, el $38 \%$ la tienen desarrollada mientras que un $5 \%$ la tienen medianamente desarrollada.

Así mismo, en la competencia de conocimiento y aplicación de los lineamientos de política pública 
para la gestión de instituciones educativas, el $38 \%$ la tienen muy desarrollada y el $62 \%$ la tienen desarrollada. Ahora, en la orientación para la toma de decisiones con base en el análisis de información proveniente de diferentes fuentes, con el fin de cumplir las metas establecidas, el $33 \%$ la tienen muy desarrollada, el $52 \%$ la tienen desarrollada y existe un porcentaje significativo del $14 \%$ que la tienen medianamente desarrollada. En esta línea, la competencia de seguimiento a la convivencia escolar teniendo en cuentas los lineamientos de política pública y el manual de convivencia, el $38 \%$ la tienen muy desarrollada, el $48 \%$ desarrollada y el $14 \%$ medianamente desarrollada.

En relación a las competencias para anticipar y proyectar los procesos de gestión de las organizaciones educativas, la competencia de decisión sobre las estrategias más convenientes para atender asuntos relacionados con la gestión de los recursos humanos de la institución, el $43 \%$ de los directivos expresa tenerla muy desarrollada, otro 43 $\%$ dice tenerla desarrollada y el $14 \%$ sostiene que la tiene medianamente desarrollada. Por su parte, la competencia de reconocimiento de los posibles resultados de diferentes cursos de acción y decisiones del Gobierno Escolar, teniendo en cuenta las características de dichas intervenciones, el $29 \%$ considera que la tiene muy desarrollada, el $62 \%$ cree tenerla desarrollada y el $10 \%$ la tiene medianamente desarrollada.

En este mismo sentido, en cuanto a la competencia de formulación de estrategias para mejorar los procesos administrativos de la institución con el fin de apoyar los procesos de enseñanza y aprendizaje, el $48 \%$ la tiene muy desarrollada, el $47 \%$ desarrollada y el $5 \%$ medianamente desarrollada. Y la competencia de la gestión y consecución de recursos físicos y humanos dentro y fuera de la institución, el $43 \%$ la tiene muy desarrollada, otro 43 $\%$ desarrollada y el $14 \%$ considera tenerla medianamente desarrollada.

En términos de las competencias para monitorear y evaluar los procesos en las organizaciones educativas, en cuanto a la competencia de evaluación del impacto de las acciones realizadas para establecer vínculos de cooperación con entidades y actores externos a la institución educativa, el $24 \%$ dice tenerla muy desarrollada, un $52 \%$ cree que la tiene desarrollada y un $24 \%$ sostiene tenerla medianamente desarrollada. Por otro lado, la evaluación del uso de los recursos institucionales para maximizar la eficacia y eficiencia de los procesos institucionales, el $52 \%$ expresa tenerla muy desarrollada y el $48 \%$ considera que la tiene desarrollada.

En cuanto al seguimiento a la convivencia escolar teniendo en cuentas los lineamientos de política pública y el manual de convivencia, el $28 \%$ sostiene tenerla muy desarrollada, el $67 \%$ la tiene desarrollada y el $5 \%$ expresa tenerla medianamente desarrollada. Por último, en la competencia de seguimiento a la gestión del personal docente y administrativo a su cargo con el fin de mejorar los resultados y lograr las metas, el $25 \%$ la tiene muy desarrollada, el $70 \%$ la tiene desarrollada y el $5 \%$ medianamente desarrollada.

En la figura 2 se muestran los niveles promedio de cumplimiento de las competencias asociadas con la planeación de la práctica educativa escolar, el desarrollo de la práctica educativa escolar, el seguimiento y mejora de la práctica educativa, la orientación al logro, la sensibilidad interpersonal, la comunicación asertiva, el liderazgo, el trabajo en equipo y la autoeficacia. 
Figura 2. Desarrollo de competencias para la gestión educativa

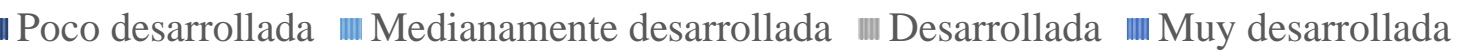

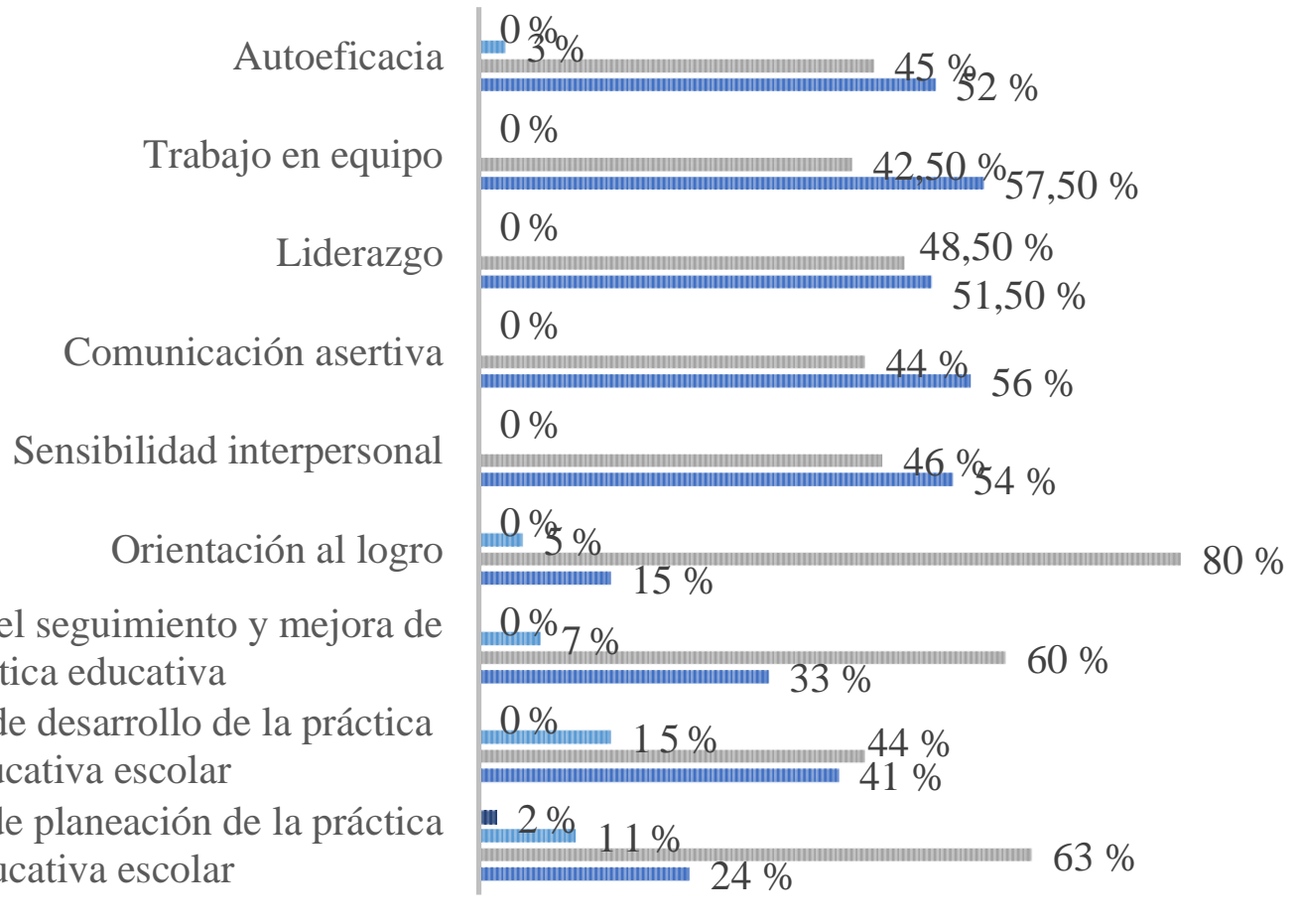

Fuente: elaboración propia

En términos generales, los niveles de las competencias son desarrollados o muy desarrollados en cada uno de los

El trabajo en equipo corresponde al componente con la mejor evaluación en cuanto a las valoraciones de la escala, mientras que la orientación al logro es la que mayor puntaje ha obtenido en el punto de desarrollada. En este sentido, es evidente que la gestión de las instituciones educativas se desarrolla pensando en generar respuestas efectivas a indicadores definidos por el sistema.

\section{DISCUSIÓN}

\section{Reflexiones sobre la transformación de las instituciones educativas en organizaciones escolares inteligentes}

Senge (1995) y Puentes (2005) coinciden en identificar la organización inteligente como aquella que posee la capacidad de aprender constantemente y reinventarse para responder a las exigencias del contexto. Al respecto, Senge (1995) señala que las organizaciones inteligentes se pueden definir como aquellas que tienen "auténtica capacidad de aprendizaje, aptas para perfeccionar continuamente su habilidad para alcanzar sus mayores aspiraciones" (p. 15). De esta forma, una organización es inteligente en la medida que sus miembros aprenden y acuden a prácticas que impulsan el mejoramiento continuo, que interactúan por el bien colectivo. Por su parte, Puentes (2005) define la organización escolar inteligente como aquella que;

Enfatiza en la importancia del aprendizaje más que de la enseñanza y cuyo propósito fundamental está estrechamente vinculado, además, con la capacidad de la escuela para generar relaciones humanas de calidad entre individuos que pueden negociar significados, propiciar la satisfacción de sus necesidades básicas y construir ambientes, creativos, autónomos y democráticos (p. 10). 
Indudablemente, una concepción de esta naturaleza para una instancia educadora otorga a la escuela un nuevo significado, porque permite el desarrollo de una organización sistémica y un mejor desempeño e interacción entre la gestión académica adelantada en las aulas y los procesos liderados por el directivo docente desde cada una de las áreas de gestión.

\section{El capitán del viaje...El líder, el rector(a)}

El viaje orientado a la resignificación de la organización educativa requiere del replanteamiento del tradicional concepto de líder. La nueva organización escolar requiere de un líder que como lo señala Maxwell (1996):

[...] una persona que guía a otra hacia una meta en común, mostrando el camino y creando un ambiente que permita la participación activa de todos los miembros del equipo. El líder es una persona comprometida a llevar a cabo un proyecto (p. 136).

Haciendo referencia a la anterior definición, comprendemos que el rector (a) como líder debe orientar su equipo de trabajo hacia el logro de los resultados concertados y definidos con anterioridad, por tanto, debe conocer las potencialidades y ser un constructor de acuerdos y de consensos.

Se considera que entre las alternativas de cambio educativo y modernización de la escuela para la conversión de la institución educativa en una organización escolar inteligente es de particular importancia, desde luego, la comprensión de este cambio y el inicio de actividades para el desarrollo de esta especie de viaje como una de las tareas que deben emprender los directivos. La organización inteligente tiene como elemento esencial el ser una estructura integrada, que trabaja como un todo, que posee valores, hábitos, políticas, programas, sistemas que apoyan y aceleran el aprendizaje organizacional y el de todos. Estos elementos existen en sus estructuras tanto formales como informales (Viloria, Daza y Pérez, 2016; Viloria, Pedraza, Cuesta y Pérez, 2016).

\section{Liderazgo y trabajo en equipo como pilares del cambio}

Como lo comenta Maxwell (AÑO), los grandes desafíos demandan un gran trabajo de equipo, en el cual cada miembro del equipo debe tener plena conciencia de su responsabilidad frente al compromiso de cambio y el hecho de alcanzar las metas más allá de lo que el líder le asignó como función en su desempeño. Así, todos y cada uno participan del cumplimiento de tareas, evalúan su proceso y miran con actitud reflexiva el accionar de la organización escolar y de cada uno de sus componentes de gestión de forma permanente hasta que este hecho, se convierte en un elemento constitutivo de la cultura organizacional. Al respecto, Correa (2005) señala que los

[...] agentes de la organización serán más eficientes cuando abandonen la exclusiva ilusión del control y el poder y se orienten por una relación comunicativa más centrada en la cooperación. Practicar la Máxima del dominio generativo, en donde se tiene menos control de lo que se quisiera, pero más influencia para la producción de la esperada (p. 112).

Efectivamente, en este campo el reto que tiene cada directivo, comprometido en el desarrollo de esta perspectiva de cambio, consiste en que a través de su liderazgo los miembros de su comunidad dejen de pensar de manera individualizada y empiecen a pensar en el nosotros, como alternativa que posibilita la consecución de metas trazadas.

Autores como Senge (1995) manifiestan que "La disciplina del aprendizaje en equipo comienza con el diálogo, la capacidad de los miembros del equipo para suspender los supuestos e ingresar en un auténtico pensamiento conjunto" (p. 19). Por consiguiente, solo a través de un trabajo en equipo realmente colaborativo y comprometido se puede concretar el discurso en acciones efectivas, empleando instrumentos y metodologías viables que fortalezcan la gestión académica como eje fundamental de la organización educativa.

\section{El nuevo horizonte de la escuela como}




\section{organización escolar inteligente}

Así, la escuela concebida como organización debe ser analizada desde una perspectiva inter, multi y transdiciplinaria, lo que ayudará a mirarla de una forma integral. La escuela como organización inteligente permitirá una mejor interacción entre sus partes y con el entorno. El nuevo horizonte, entonces, se entiende como un camino de pequeños cambios que de manera paulatina repercutirán en una gran transformación. No se puede pretender cambiar la escuela de la noche a la mañana, porque esta no es el edificio donde ocurre la formación como muchas veces el común de las personas pueden definir, la escuela son las personas y los procesos que se llevan a cabo en ella. Por consiguiente, se requiere un diagnóstico previo de la situación, así como la contextualización para iniciar el camino al cambio y una permanente evaluación de las acciones para reforzar conductas positivas, porque la transición para convertirse en una organización escolar inteligente no se puede hacer bajo presión y sin contar con el cambio de cada uno de los actores. Es necesario fortalecer su orientación al logro, entendido este como el esfuerzo que los miembros de un equipo realizan, de forma individual y colectiva, para cumplir los objetivos trazados dentro de las expectativas planteadas.

En consecuencia, vista la escuela a través de este enfoque se logrará estudiar el sistema de la organización en sí mismo, reconociendo cómo se entrelazan y afectan de manera recíproca todos los componentes de gestión que interactúan a través del tiempo en el escenario escolar, en aras de conseguir un mismo fin, es así como se logrará definir la problemática, hallar oportunidades y conseguir las soluciones.

Por lo anterior el rector(a) como líder debe procurar que todos los miembros de la comunidad tengan una visión unificada y reconozcan el papel que desempeñan dentro de este sistema que opera como un todo y no como partes separadas, también, debe promover un proceso permanente de reconocimiento de la organización escolar, en el que se estudien las situaciones presentadas que impiden el progreso, las analice con su equipo, promueva el diseño de estrategias de mejora, estimule la puesta en práctica a través de una adecuada implementación de estas propuestas y las evalué para que se puedan mantener en el tiempo y contribuyan de manera efectiva en los procesos de mejora continua.

Al concebir que la escuela es un sistema se reconoce que existen diferentes elementos que la conforman y hacen que funcione. Si bien no podemos desconocer que el trabajo en el aula es transcendental en el quehacer, en las metas trazadas por la escuela y dentro del proceso de la gestión académica, debemos entender también que componentes como la gestión Administrativa-Financiera, Directiva y Comunitaria, inciden con notoriedad en que los fines de la organización escolar se alcancen. Si deseamos una organización realmente inteligente debemos traspasar las fronteras materiales impuestas y lograr que los aprendizajes trasciendan más allá del aula de clases, ya que las teorías de la inteligencia se apliquen en los diversos contextos organizacionales (Cabas, González y Hoyos, 2017).

En esta nueva perspectiva se invita a los rectores(as) a focalizar el trabajo de su organización hacia los procesos ejecutados con miras al desarrollo de competencias para la gestión académica, el mejoramiento del currículo y el fortalecimiento del liderazgo pedagógico. En este sentido, se requiere una gestión educativa cuyo cimiento sea el trabajo de todos los miembros de la comunidad, quienes deben estar comprometidos y dispuestos a cooperar entre sí para solucionar conflictos, superar retos y aprender, porque existe al respecto, la plena conciencia que a través de la innovación y el flujo permanente de iniciativas que permitan el logro de los objetivos institucionales se puede ir más allá de un currículo preestablecido y reconocer la responsabilidad social que tiene la organización.

\section{CONCLUSIÓN}

Al concebir la institución educativa como una organización escolar inteligente, estamos ante un nuevo constructo integrado por elementos pedagógicos, administrativos y organizacionales, lo que será fundamental en la orientación del cambio, pero sobre todo en la concreción de éste en la realidad 
de los escenarios educativos. Esta transformación y las nuevas organizaciones escolares deben estar orientadas por el rector, convertido ahora en un líder efectivo que reconoce la importancia de su liderazgo como transformador de la realidad institucional y le abre un mundo de posibilidades nuevas para fortalecer el trabajo con su comunidad.

Los Rectores y coordinadores, como líderes de la gestión directiva, deben contar con las herramientas de formación necesarias que les permitan responder a los retos establecidos en los nuevos modelos de gestión que exigen las organizaciones escolares, están llamados a incentivar en los maestros a un mayor compromiso educativo y esto solo será posible, a través de la construcción de una visión compartida y una misión de compromiso que cimienten el horizonte institucional, por tanto es tarea de las Secretarias de Educación, en asocio con las universidades, diseñar programas de formación que den cuenta de las necesidades, expectativas y requerimientos que realmente respondan a lo que exigen los contextos educativos actuales.

Esto es, a través de programas de formación Profesional permanente para los directivos docentes que partan de la caracterización del perfil profesional de estos y de las competencias que tienen para el desarrollo de la gestión educativa. Donde trascienda la simple adquisición de conceptos teóricos y se entreguen bases epistemológicas para construir un concepto de lo que es la gestión educativa y cuáles son los requerimientos para desarrollarla de manera eficiente.

Se debe promover acciones de formación orientadas de manera puntual a: Fortalecer el Liderazgo de la gestión educativa, para que se desarrolle de manera compartida, involucrando a todos los miembros de la comunidad, brindando las herramientas conceptuales para Resignificar el Proyecto Educativo Institucional (PEI), así como la organización de los sistemas de información, evaluación y seguimiento de procesos.

En este orden de ideas, se hace pertinente además la actualización de conocimientos en lo relacionado con el currículo, modelos pedagógicos, formulación de proyectos y la investigación como componente innegable de los cambios orientados hacia la mejora de la calidad institucional.

Por otra parte, en cuanto a la administración de recursos, se debe contar con el sustento para ser capaz de promover el desarrollo integral del personal, así como la optimización del gasto, para que la gestión se encuentre dentro del marco de la legislación establecida en Colombia, partiendo de un reconocimiento e interpretación adecuado de la ley. Sin desconocer como aspecto importante lo relacionado con la promoción de espacios de formación ciudadana, en el marco de los valores y la sana convivencia.

Es válido, por consiguiente, reconocer que el progreso y consolidación de muchas organizaciones se debe en una gran parte a la responsabilidad de los líderes que las orientan, quienes a través del conocimiento de las potencialidades y limitaciones de los miembros de la conforman y el reconocimiento de su contexto en el cual se encuentran inmersos, son capaces de trazar metas de interés colectivo que propendan en la consecución del mejoramiento progresivo. Es así como se visiona al directivo docente, como actor principal en el liderazgo de procesos de cambio en la transformación de las organizaciones escolares a través de su experiencia profesional y cualidades personales.

\section{Declaración sobre conflicto de interés}

Los autores declaran que no existe conflicto de intereses en la investigación. Así mismo, que los resultados y afirmaciones académicas expuestas se desprenden del proceso investigativo y no han sido influenciadas por ninguna persona o institución.

\section{REFERENCIAS BIBLIOGRÁFICAS}

Aguerrondo, I. (1998). La escuela como organización inteligente. Argentina: Troquel Editorial.

Cabas, H. K., González, B. Y. y Hoyos, R. P. (2017). Teorías de la inteligencia y su práctica en el 
COMPETENCIAS DE LOS DIRECTIVOS DOCENTES PARA LA TRANSFORMACIÓN DE LAS INSTITUCIONES EDUCATIVAS EN ORGANIZACIONES ESCOLARES INTELIGENTES

siglo XXI: Una revisión. Clío América, 11(22), 254-270. Recuperado de http://revistas.unimagdalena.edu.co/index.php /clioamerica/article/view/2445

Correa, A., Álvarez, A. y Correa, S. (2012). La Gestión Educativa: un nuevo paradigma. Medellín: Fundación Universitaria Luis Amigó. Recuperado de http://virtual.funlam.edu.co/repositorio/sites/d efault/files/6lagestioneducativaunnuevoparadi gma.pdf

Correa, C. (2005). Administración estratégica y calidad integral en las instituciones educativas. Bogotá, Colombia: Editorial Magisterio.

Daza, A., Miranda, L. y Rodríguez, F. (2017). Gerencia del talento humano como estrategia potenciadora de la calidad del servicio educativo en universidades públicas Anotaciones desde una perspectiva teórica. En Herrera, D., Ramírez, G., y Rosas, J. (Ed), Diversidad y Complejidad Organizacional en América Latina (pp. 253 - 286). Ciudad de México, México: Grupo Editorial Hess. Recuperado de http://remineo.org/repositorio/libros/dcoal/wp -content/uploads/2017/08/08-La-calidadeducativa.pdf\#page $=253$

Drucker, P. (2012). The practice of management. New York: Routledge.

González, A. (2006). Métodos de compensación basados en competencias. Barranquilla, Colombia: Ediciones Uninorte. Recuperado de https://books.google.com.co/books?id=v3qcle $\underline{\mathrm{mGtvkC} \& \text { printsec }=\text { frontcover } \& \mathrm{hl}=\mathrm{es} \& \text { source }}$
$=$ gbs ge summary $\mathrm{r} \& \mathrm{cad}=0 \# \mathrm{v}=$ onepage $\& \mathrm{q} \&$ $\underline{\mathrm{f}=\mathrm{false}}$

González, E. (2017). Gestión de la identidad pedagógica organizacional: En busca de coherencia y unidad de la intencionalidad formativa. En Herrera, D., Ramírez, G., y Rosas, J. (Ed), Diversidad y Complejidad Organizacional en América Latina (pp. 51 92). Ciudad de México, México: Grupo Editorial Hess. Recuperado de http://remineo.org/repositorio/libros/dcoal/wp -content/uploads/2017/08/01-Vida-

\section{$\underline{\text { Simbolica.pdf\#page }=51}$}

Hernández, R., Fernández, C. y Baptista, P. (2010). Metodología de la investigación. México: Editorial Mc Graw Hill. Recuperado de file://C:/Users/revistaclioamerica/Downloads/ _Hernandez_Sampieri_R._Fernandez_Collad. pdf

Higuita, D., Molano, J. y Rodríguez, M. (2011). Competencias necesarias en los grupos de investigación de la Universidad Nacional de Colombia que generan desarrollos de base tecnológica. Innovar 21(41), 209-224. Recuperado de https://revistas.unal.edu.co/index.php/innovar/ article/view/35405/35777

Liñán, C. y Polo, M. (2017). La complejidad administrativa de las instituciones públicas de educación básica y media en Colombia: Una mirada crítica al contexto. En Herrera, D., Ramírez, G., y Rosas, J. (Ed), Diversidad y Complejidad Organizacional en América Latina (pp. 119 - 152). Ciudad de México, México: Grupo Editorial Hess. Recuperado de 
http://remineo.org/repositorio/libros/dcoal/wp -content/uploads/2017/08/09-Practicas-yrealidades.pdf\#page $=119$

Lombana, C. J. y Muñoz, S. A. (2017). Competitividad, educación y empleabilidad: consideraciones para el desarrollo de los países. Clío América, 11(22), 169-176. Doi: $10.21676 / 23897848.2437$

Maxwell, J. (1996). Desarrolle el Líder que está en usted. EE. UU: Editorial Caribe. Recuperado de

https://www.webdelclub.com/whuracan/docu/ des000019.pdf

Maxwell, J. (2001). Las 17 cualidades esenciales de un jugador de equipo. EE. UU: Grupo Nelson.

Murcia, N. y Jaramillo, L. (2000). La investigación cualitativa. La investigación etnográfica. Armenia, Colombia: Kinesis.

Ocampo, J., Castro, W., Becerra, G. y Herrera, B. (2014). Caracterización del perfil del docente de los Programas de Administración y sus competencias para la docencia. Bogotá D.C.: Trazos y Diseños H y D. Recuperado de http://www.ascolfa.edu.co/web/archivos/LIB RO CARACTERIZACION DEL PERFIL DEL_DOCENTE_DE_LOS_PROGRAMAS DE_ADMINISTRACION.pdf

Ortiz, O. A. (2015). Enfoque y Métodos de Investigación en las Ciencias Sociales $y$ Humanas. Bogotá: Ediciones de la U.

Peralta, E. (2016). Teoría general de los sistemas aplicada a modelos de gestión. Aglala, 7(1), 122-145. Recuperado de http://revistas.curnvirtual.edu.co/index.php/ag

\section{lala/article/view/901}

Perkins, D. (1995). Escuela inteligente (Vol. 17). Barcelona: Gedisa.

Perkins, D. (2000). Schools need to pay more attention to 'intelligence in the wild'. Harvard Education Letter, 16(3), 1-3.

Polo, M. y Liñán, C. (2017). El proyecto educativo institucional-PEI: Una mirada desde la gestión directiva. En Herrera, D., Ramírez, G., y Rosas, J. (Ed), Diversidad y Complejidad Organizacional en América Latina (pp. 83 118). Ciudad de México, México: Grupo Editorial Hess. Recuperado de http://remineo.org/repositorio/libros/dcoal/wp -content/uploads/2017/08/09-Practicas-yrealidades.pdf\#page $=83$

Puentes, Y. (2005). Organizaciones escolares inteligentes educativas. Bogotá. Colombia: Editorial Magisterio.

Rodríguez, Á. (2017a). Las concepciones del liderazgo educativo a partir de las voces de los actores sociales de la gestión educativa en la organización escolar. En Herrera, D., Ramírez, G., y Rosas, J. (Ed), Diversidad y Complejidad Organizacional en América Latina (pp. 223 260). Ciudad de México, México: Grupo Editorial Hess. Recuperado de http://remineo.org/repositorio/libros/dcoal/wp -content/uploads/2017/08/09-Practicas-yrealidades.pdf\#page $=223$

Rodríguez, Á. (2017b). Praxis de liderazgo en la gestión educativa de la organización escolar: Una mirada reflexiva desde los directivos docentes y docentes. En Herrera, D., Ramírez, 
COMPETENCIAS DE LOS DIRECTIVOS DOCENTES PARA LA TRANSFORMACIÓN DE LAS INSTITUCIONES EDUCATIVAS EN ORGANIZACIONES ESCOLARES INTELIGENTES

G., y Rosas, J. (Ed), Diversidad y Complejidad

Organizacional en América Latina (pp. 261 302). Ciudad de México, México: Grupo Editorial Hess. Recuperado de http://remineo.org/repositorio/libros/dcoal/wp -content/uploads/2017/08/09-Practicas-y$\underline{\text { realidades.pdff } \# \text { page }=261}$

Sánchez, J. (2010). Hacia un paradigma emergente de la planeación: Resignificación de las instituciones universitarias. Santa Marta, Colombia: Editorial Unimagdalena.

Sánchez, J. (2014). Prácticas de Autoevaluación con fines de Acreditación Institucional en Colombia: una lectura evaluativa $y$ propositiva desde la perspectiva de la construcción social de la realidad. Santa Marta, Colombia: Editorial Unimagdalena.

Sánchez, J., Chica, O. y Viloria, J. (2017). Prácticas de gestión directiva y académica que interpelan de manera negativa la calidad de las instituciones educativas públicas: El caso del Distrito de Santa Marta, Colombia. En Herrera, D., Ramírez, G., y Rosas, J. (Ed), Diversidad y Complejidad Organizacional en América Latina (pp. 55 - 92). Ciudad de México, México: Grupo Editorial Hess. Recuperado de http://remineo.org/repositorio/libros/dcoal/wp -content/uploads/2017/08/08-La-calidadeducativa.pdf\#page $=55$

Sánchez, J., Linero, D. y Martínez, M. (2014). Ocultamiento del discurso pedagógico frente al discurso administrativo en la gestión de las organizaciones educativas. Clío América, 8(15), 36-46. Recuperado de http://revistas.unimagdalena.edu.co/index.php /clioamerica/article/view/828

Sánchez, J., Sánchez, I. y Viloria, J. (2017). Expectativas de formación avanzada en el campo de la gestión educativa: una lectura desde las voces de directivos docentes en el departamento del Magdalena, Colombia. En Herrera, D., Ramírez, G., y Rosas, J. (Ed), Diversidad y Complejidad Organizacional en América Latina (pp. 21 - 60). Recuperado de http://remineo.org/repositorio/libros/dcoal/wp -content/uploads/2017/08/12-Territorio-yorganizaciones.pdf\#page $=21$

Sánchez, J., Viloria, J. y Miranda, L. (2017). Los grupos informales en las organizaciones escolares: un acercamiento desde la gestión educativa. Praxis, 13(1), 56-68. Recuperado de http://revistas.unimagdalena.edu.co/index.php /praxis/article/view/2068

Senge, P. (1990). La quinta disciplina. Cómo impulsar el aprendizaje en la organización inteligente. Buenos Aires, Argentina: Granica/Vergara.

Senge, P. (1995). La Quinta Disciplina. Buenos Aires: Granica.

Silva, G. H. (2017). La innovación y educación: variables claves para la competitividad de las empresas. Clío América, 11(21), 88 -107. Doi: http://dx.doi.org/10.21676/23897848.2079

Torres, C. M., Vélez, P. J. y Altamar, B. F. (2015). La calidad de la educación superior en Colombia. Una aproximación econométrica (2007 2012). Clío América, 9(18), 143 - 156. Recuperado de http://revistas.unimagdalena.edu.co/index.php /clioamerica/article/view/1532

Viloria, J., Bertel, M. y Daza, A. (2015). Percepciones 
estudiantiles sobre el proceso de acreditación por alta calidad del Programa de Administración de Empresas de la Universidad del Magdalena. Praxis, 11, 89-102. Recuperado de http://dx.doi.org/10.21676/23897856.2068

Viloria, E. J, Pedraza, A. L, Cuesta, T. K. y Pérez, C. K. (2016). Liderazgo informal en las organizaciones: reflexiones sobre su impacto e influencia en la competitividad. Clío América.
10(19), $31 \quad-\quad 42 . \quad$ Recuperado de http://revistas.unimagdalena.edu.co/index.php /clioamerica/article/view/1658

Viloria, J., Daza, A. y Pérez, K. (2016). Dinámicas e influencias de los grupos informales en las organizaciones. Ánfora, 23(40), 169-194. Recuperado de http://publicaciones.autonoma.edu.co/index.p hp/anfora/article/view/9/7 